$$
f=\left(1+T / 3 t^{*}\right)^{-1} .
$$

The differential equation ${ }^{3,4}$ used hitherto for a radiation field of energy density $u$ is

$$
d\left(u R^{4}\right) / d t=R^{4}\left(u^{*}-u\right) / \tau,
$$

and the condition for darkness at night in a steady-state universe is hence

$$
u / u^{*}=T /(4 \tau+T) \ll 1,
$$

which is the well-known result quoted by Lategan.

The first term on the right of Eq. (3) represents the rate of emission by luminous sources, and the second term represents the rate of absorption by luminous sources only. Since sources exist that have decayed and become nonluminous, the second term must include absorption by both luminous and nonluminous sources, and the differential equation should therefore take the form

$$
d\left(u R^{4}\right) / d t=\left(R^{4} / \tau\right)\left(u^{*}-u / f\right) .
$$

The condition for darkness thus becomes

$$
u / u^{*}=T f /(4 \tau f+T) \ll 1
$$

and is dependent, through $f\left(t^{*}, T\right)$, on the luminous lifetime $t^{*}$. It is now evident that a sufficient condition for darkness is $f \ll 1$, or $3 t^{*} \ll T$, and this is also a necessary condition in those steady-state universes of $T^{2} \gg 12 \pi t^{*}$. When $T^{2} \ll$ $12 \tau t^{*}$, the sufficient and necessary condition is $T \ll 4 \tau$, as before.

The above argument, I think, confirms my previous belief that the luminous lifetime $t^{*}$ cannot be ignored in the steady-state universe. Also, we use "growth time" and "decay time" for exponential functions, and I do not think the expression "expansion time" is misleading when applied to the steady-state universe.

'E. R. Harrison, Phys. Today 27, 30 (1974).

${ }^{2}$ E. R. Harrison, Am. J. Phys. 45, 124 (1977).

${ }^{3}$ E. R. Harrison, Mon. Not. R. Astr. Soc. 131, 1 (1965).

${ }^{4}$ E. R. Harrison, Vistas in Astron. 20, 341 (1977).

\title{
Widespread error in a standard problem in the dynamics of deformable bodies
}

\author{
Juan R. Sanmartin and Miguel A. Vallejo \\ Cátedra de Física, E. T. S. I. Aeronáuticos, Universidad Politécnica de Madrid, Spain \\ (Received 7 January 1977; accepted 28 March 1978)
}

While recently discussing variable mass systems, Siegel ${ }^{1}$ considered a standard problem in the mechanics of deformable bodies: A homogeneous chain (or flexible, inextensible rope), of length $L$ and mass $\lambda L$, slides off a smooth table, starting from rest with a length $\alpha L(\alpha<1)$ hanging over the table (see Fig. 1). Siegel gives

$$
\ddot{X}_{A}=g X_{A} / L
$$

as the equation of motion for the entire range $\alpha L \leq X_{A} \leq$ $L$; this equation implies the arrangement of Fig. 1 .

We wish to point out first that actually Eq. (1) becomes invalid, the chain separating from the corner, before end $B$ leaves the table. Indeed, if separation would not occur, momentum along the $y$ axis would vanish when either $X_{A}$ $=\alpha L$ or $X_{A}=L$, and would be positive in between, decreasing during part of the motion; but this is obviously impossible since the $y$ component of the reaction at the corner cannot be negative. Equation (1) is (wrongly) used

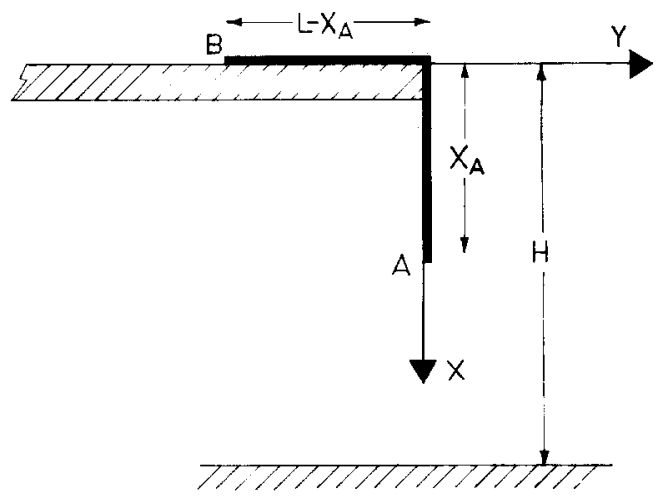

Fig. 1. Chain of length $L$ and links of negligible size, sliding off a smooth table. in a variety of textbooks and treatises ${ }^{2-11}$ to determine either the time when B leaves the table or $\dot{X}_{A}$ at that time. The need to externally guide the chain around the corner to avoid separation is noticed by Meriam ${ }^{12}$ and Greenwood. ${ }^{13,14}$ The falling chain problem is considered in many other texts with no mention of the range of validity of Eq. (1).

A proof that separation does occur, even if friction exists on both table top and corner, is given by Lainé. ${ }^{15} \mathrm{~A}$ simpler proof is as follows: As long as there is no separation, the equations of motion for the shaded parts drawn in Fig. 2(a) are

$$
\begin{gathered}
T_{2}-\mu \lambda\left(L-X_{A}\right) g=\lambda\left(L-X_{A}\right) \ddot{X}_{A}, \\
\lambda X_{A} g-T_{1}=\lambda X_{A} \ddot{X}_{A},
\end{gathered}
$$

while at the corner [Fig. 2(b)]

$$
\frac{\partial T}{\partial \phi}=-\mu N+\lambda \rho g \cos \phi-\lambda \rho \ddot{X}_{A},
$$

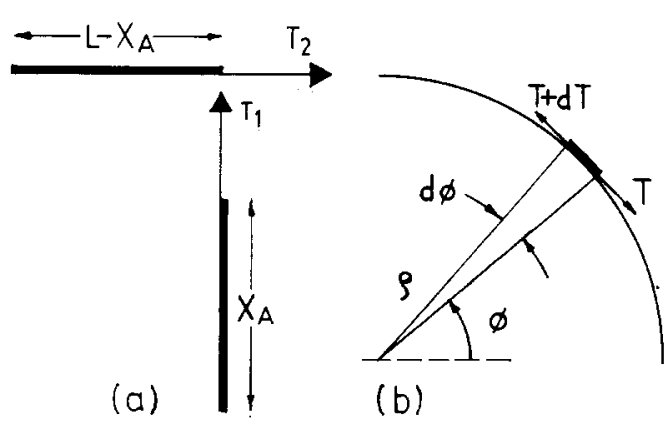

Fig. 2. Motion of the various chain pieces. (a) Horizontal and vertical pieces. (b) Arc of chain at the corner; $\rho$ is the radius of curvature. 


$$
N=T-\lambda \dot{X}_{A}^{2}+\lambda \rho g \sin \phi,
$$

$\mu$ being the friction coefficient and $N$ the normal reaction per unit angle; separation will occur if $N$ becomes negative. Letting $\rho \rightarrow 0$, Eqs. (4) and (5) yield

$$
\begin{gathered}
T(\phi)=\lambda \dot{X}_{A}^{2}+\left(T_{1}-\lambda \dot{X}_{A}^{2}\right) \exp (-\mu \phi), \\
N(\phi)=T(\phi)-\lambda \dot{X}_{A}^{2} .
\end{gathered}
$$

Assume that $N(\phi)$ is non-negative throughout and consider the time when $X_{A}=L$. Equation (2) gives $T_{2} \equiv T(\pi / 2)$ $=0$, and therefore $\dot{X}_{A}=0$ for $N(\pi / 2)$ to be non-negative. It follows from Eq. (6) that $T_{1}=0$, and then Eq. (3) leads to $\ddot{X}_{A}=g$. But clearly we cannot have both $\dot{X}_{A}=0$ and $\ddot{X}_{A}$ $>0$ if the chain is falling!

We find that a chain sliding off a table with a round corner ( $\rho$ comparable to $L$ ) does also separate. Otherwise, Eqs. (3)-(5) would remain valid when $X_{A}=L$ (that is, when $B$ reaches the end of the corner at $\phi=0$ ); then $T_{1}$ $\equiv T(0)=0$ and, from Eqs. (3) and (5), we recover the incompatible results $\ddot{X}_{A}=g, \dot{X}_{A}=0$.

Motion after separation is extremely complex. However, for small $L / 2 H, H$ being the table height, we may find immediately the time of fall $t_{f}$, to order $(L / 2 H)^{1 / 2}$

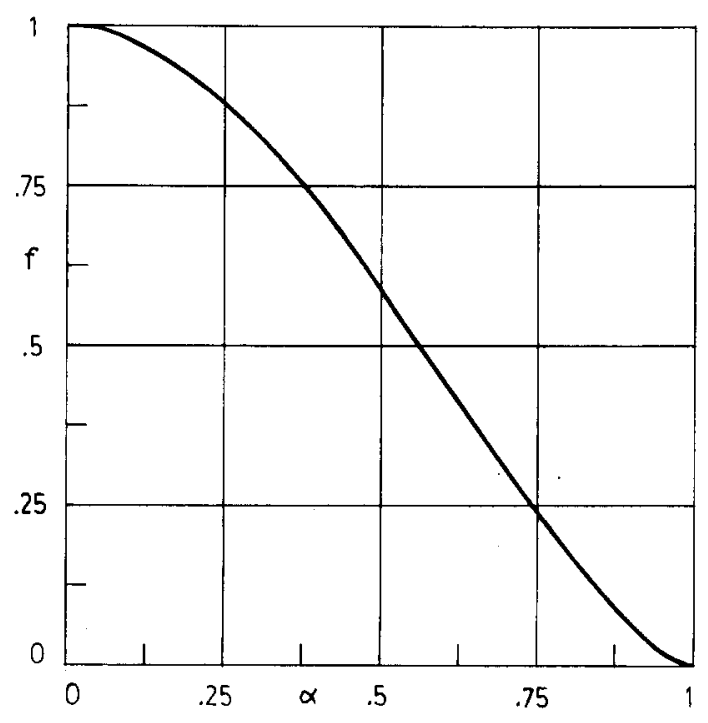

Fig. 3. Dimensionless factor $f(\alpha)$ for the horizontal distance traveled by the chain during the fall.

$$
t_{f}=(2 H / g)^{1 / 2}
$$

Then, if $V_{y}$ is the velocity, along the $y$ axis, of the chain center of mass, we have

$$
Y_{f}=V_{y}^{*} t_{f}=\left[\left(L-X_{A}^{*}\right) \dot{X}_{A}^{*} / L\right] t_{f}
$$

as the horizontal distance traveled by the chain during the fall (* labeling separation conditions). From Eqs. (2), (3), $(6)$, and (7), neglecting friction for small $\mu$, we get $X_{A}^{*}$ and $\dot{X}_{A}^{*}$ by setting $N=0$. Then

$$
\begin{gathered}
Y_{f}=(H L / 8)^{1 / 2} f(\alpha)\left\{1+0(\mu)+0\left[(L / 2 H)^{1 / 2}\right]\right\} \\
f(\alpha) \equiv\left\{\left[3-\left(1+8 \alpha^{2}\right)^{1 / 2}\right] / 2\right\} \\
\times\left\{\left[1-4 \alpha^{2}+\left(1+8 \alpha^{2}\right)^{1 / 2}\right] / 2\right\}^{1 / 2}
\end{gathered}
$$

Figure 3 shows $f(\alpha)$. For $H=2 \mathrm{~m}, L=4 \mathrm{~cm}, \alpha \ll 1$ we obtain $Y_{f}=10 \mathrm{~cm}$.

'S. Siegel, Am. J. Phys. 40, 183 (1972).

2P. Appell, Traité de Mécanique Rationnelle (Gauthier-Villars, Paris, 1931), Vol. II, pp. 43-44; Précis de Mécanique Rationnelle (Gauthier-Villars, Paris, 1952), pp. 341-342.

${ }^{3} \mathrm{~K}$. Wolf, Lehrbuch der Technischen Mechanik Starrer Systeme (Springer-Verlag, Vienna, 1947), pp. 297-299.

${ }^{4} \mathrm{~S}$. Timoshenko and D. H. Young, Engineering Mechanics (McGrawHill, New York, 1956), p. 308.

${ }^{5}$ I. H. Shames, Engineering Mechanics. Statics and Dynamics (Prentice-Hall, Englewood Cliffs, NJ, 1961), p. 444.

${ }^{6} \mathrm{~B}$. Finzi and P. Udeschini, Esercizi di Meccanica Razionale (Tamburini, Milan, 1962), pp. 438-439.

${ }^{7}$ R. R. Long, Engineering Science Mechanics (Prentice-Hall, Englewood Cliffs, NJ, 1963), p. 197.

${ }^{8}$ M. R. Spiegel, Theoretical Mechanics (McGraw-Hill, New York, 1967), problem 3.85 .

${ }^{9} \mathrm{~A}$. Giet, Problèmes de Mécanique (Dunod, Paris, 1968), p. 254.

${ }^{10}$ E. Gullón and M. López, Problemas de Física (Librería Internacional de Romo, Madrid, 1969), p. 221.

"I. Mesherski, Problemas de Mecánica Teórica, translated by F. Petrov (MIR, Moscow, 1974), p. 379.

12J. L. Meriam, Mechanics (Wiley, New York, 1959), problems 222, 223 , 424 , and 425 .

${ }^{13}$ D. T. Greenwood, Principles of Dynamics (Prentice-Hall, Englewood Cliffs, NJ, 1965), pp. 180-181.

14J. P. Den Hartog, Mechanics (Dover, New York, 1961), p. 192, notices also the need to guide the chain. However he gives $2^{1 / 2} T$ as the reaction at the corner, while from Eqs. (6) and (7), for $\mu=0$, we get $2^{1 / 2}(T$ $\left.-\lambda \dot{X}_{A}^{2}\right)$; that is, Den Hartog neglects the very term $\lambda \dot{X}_{A}^{2}$ which makes separation possible.

${ }^{15} \mathrm{E}$. Lainé, Exercices de Mécanique (Librairie Vuibert, Paris, 1964), pp. 212-216.

\title{
Relative velocities and the runner
}

\author{
R. L. Armstrong \\ Physics Department, Box 3D, New Mexico State University, Las Cruces, New Mexico 88003 \\ (Received 1 March 1978; accepted 28 March 1978)
}

Many people today, including many science and engineering students, run for physical fitness and pleasure. I have generated interest in the study of relative velocities in our introductory physics course by asking the following question: "Suppose I am running with the wind. Through what angle must I turn so that the wind appears to me to be blowing directly from the side?" A common, intuitive (and incorrect) answer is $90^{\circ}$. The correct answer varies from "it never does" to "zero" to "an angle less than about $78^{\circ}$ " depending on whether the wind speed is less than, equal to, or greater than my running speed. An elementary application of either geometrical or vector methods will readily provide these answers.

Let $\mathbf{v}$ and $\mathbf{v}^{\prime}$ be my velocity relative to the ground and to the wind, respectively, and let $u$ be the wind velocity, with $\mathbf{v}=\mathbf{u}+\mathbf{v}^{\prime}$. Note that $I$ am sensitive to the apparent wind 